\title{
STRUCTURE DES NOYAUX LÉGERS
}

\author{
par J. Yoccoz \\ Centre de Recherches Nucléaires, Département de Physique Théorique, Strasbourg-Cronenbourg (France)
}

\begin{abstract}
Résumé. - Nous examinons les aspects actuels de l'étude de la structure des noyaux légers et nous analysons, au vu des contributions récentes, les problèmes relatifs aux choix des fonctions d'onde et énergies individuelles, de l'interaction résiduelle et des modes de couplage.
\end{abstract}

Abstract. - We review some recent developments in the understanding of the structure of light nuclei. We analyse the various ways of choosing the single particle wave functions and energies, the residual interaction and the coupling scheme.

Le modèle en couches reste la base de toute théorie dans le domaine des noyaux légers $(4 \leqslant A \leqslant 40)$. Le nombre de configurations est encore assez faible pour que des calculs raisonnables puissent être envisagés avec les gros ordinateurs, et le nombre de particules n'est pas encore assez grand pour que des schémas semi-classiques (vibrations ou rotations) puissent être utilisés brutalement. Les composantes de tout calcul dans le cadre du modèle en couches sont :

a) le choix des fonctions d'onde individuelles,

b) le choix des énergies individuelles,

c) le choix de l'interaction résiduelle,

d) le choix du couplage.

a) Le choix des fonctions d'onde individuelles. A part certains calculs dont il sera question plus tard, le potentiel moyen est supposé sphérique. Les fonctions d'onde utilisées sont en général celles d'un oscillateur harmonique, dont le paramètre $a_{0}$ (《(rayon )), est choisi de façon à avoir un rayon quadratique moyen correct pour la densité de charge. Ces fonctions ont une forme analytique simple, elles permettent une élimination simple du mouvement du centre de masse, elles donnent une densité de charge qui semble correcte et, comme de toute façon les fonctions radiales ont peu de nœuds (un seul pour $r \neq 0$ pour les fonctions $2 s$ ), la forme précise n'a peut-être pas beaucoup d'importance pour les calculs d'énergie. Cette forme, toutefois, risque d'en avoir une dans les calculs des transitions électromagnétiques de type électrique. Pour prendre un exemple, dans ${ }^{16} \mathrm{O}$ les fonctions d'onde $1 p$ et $1 s$ peuvent pratiquement être celles de l'oscillateur harmonique (cf. den- sité des charges de l'état fondamental), mais les fonctions $2 s$ et $1 d$ intervenant dans le calcul des niveaux excités correspondent à des états peu liés (ou non liés), et par conséquent doivent avoir une queue (pour les grandes valeurs de $r$ ) beaucoup plus importante que les fonctions d'onde de l'oscillateur. Les $B E$ [2] faisant intervenir les valeurs moyennes de $r^{2}$ peuvent être aussi sous-estimés par un facteur 2 ou 3 quand on utilise les fonctions d'onde oscillateur harmonique.

b) Le choix des énergies individuelles. $-1^{\circ} \mathrm{La}$ philosophie le plus souvent utilisée est celle qui consiste à tirer ces énergies de l'expérience, en supposant une configuration simple (une particule ou un trou par rapport à une couche fermée) pour des noyaux comme ${ }^{17} \mathrm{O}$ et ${ }^{17} \mathrm{~F},{ }^{15} \mathrm{O}$ et ${ }^{15} \mathrm{~N},{ }^{13} \mathrm{C}$ et ${ }^{13} \mathrm{~N}$. On a aussi, pour aider, les résultats des réactions ( $p, 2 p$ ).

Ce qui donne le schéma suivant, pour les énergies individuelles autour de ${ }^{16} \mathrm{O}$ :

$$
\begin{aligned}
& \sim 6 \mathrm{MeV} \sim 11,5 \mathrm{MeV} \sim \begin{array}{l}
0,50 \mathrm{MeV} \\
0,87 \mathrm{MeV} \sim 4,20 \mathrm{MeV}
\end{array} \\
& 1 p_{\frac{3}{2}} \quad 1 p_{\frac{1}{2}} \quad 1 d_{\frac{3}{2}} \quad 2 s_{\frac{1}{2}} \quad 1 d_{\frac{3}{2}}
\end{aligned}
$$

Par contre, la même philosophie, appliquée à ${ }^{12} \mathrm{C}$ comme cœur donne

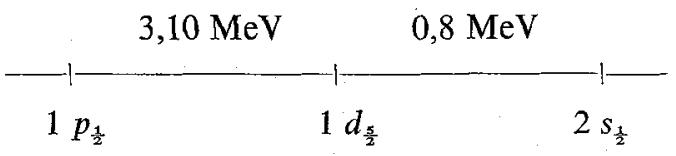

Et au-dessous de ${ }^{40} \mathrm{Ca}$, on a 


$$
\left\{\begin{array}{l}
2 s_{\frac{1}{2}}-1 d_{\frac{3}{2}}=1,22 \mathrm{MeV} \text { (Brussaard) [7] } \\
2 s_{\frac{1}{2}}-1 d_{\frac{3}{2}} \sim 1-4 \mathrm{MeV}(\mathrm{p}, 2 \mathrm{p}) \\
2 s_{\frac{1}{2}}-1 d_{\frac{5}{2}} \sim 4 \pm 1 \mathrm{MeV}(\mathrm{p}, 2 \mathrm{p})
\end{array}\right.
$$

Le calcul des spectres d'énergie, et aussi les propriétés des états correspondants, peuvent parfois être très sensibles à ce choix. Par exemple dans le calcul des probabilités de transition de ${ }^{18} \mathrm{O}$, Engeland [1] trouve qu'une variation de l'énergie de séparation entre la couche $1 p$ et $2 s-1 d$ de $1 \mathrm{MeV}$ (entre 12,5 et $13,5 \mathrm{MeV}$ ) modifie des rapports de branchement d'un facteur 3 (certains $B E$ [2] d'un facteur 6), ce qui ne laisse pas d'être inquiétant, étant donné qu'on ne peut pas être strictement sûr que des noyaux tels que ${ }^{17} \mathrm{O}$, etc... aient une structure aussi simple qu'on l'a supposé.

$2^{\circ}$ Une deuxième philosophie est de considérer ces quantités comme des paramètres, que l'on ajustera comme seront ajustés d'autres paramètres de la théorie.

$3^{\circ} \mathrm{La}$ solution idéale (au moins pour l'esprit), serait de déterminer ces énergies individuelles et, en même temps, les fonctions d'onde individuelles par un calcul de champ self-consistant Hartree-Fock, et ceci, à partir d'une interaction nucléon-nucléon aussi réaliste que possible. Il n'y a pas de succès spectaculaire dans cette voie, mais néanmoins quelques progrès qui méritent d'être relatés. Le problème essentiel est l'élimination de la partie à courte portée du potentiel qui, mal connue, infiniment répulsive ou non, introduit des corrélations à courte portée qui, de toute façon, ne peuvent pas être reproduites par des schémas tels que le modèle en couches. La structure de ces corrélations ne doit guère varier de noyau à noyau et, dans un noyau, de l'état fondamental à un état faiblement excité.

Par contre, les états faiblement excités diffèrent essentiellement par leurs corrélations à longue portée ( $>1 \mathrm{fm})$. D'où :

- Soit l'idée Scott-Moszkowski de séparer l'interaction entre nucléons en une partie à longue portée $V_{l}(\neq 0$ pour $r>d)$ et une partie à courte portée $\left(V_{s} \neq 0\right.$ pour $\left.r<d\right)$, $d$ étant choisi, en gros, de façon que $V_{s}$ n'ait pas d'influence (on compense le cœur répulsif par une certaine fraction de la partie attractive du potentiel). La façon détaillée de choisir $d$ peut varier, soit que l'on considère la diffusion libre (Kallio Kolltweit) [2], soit que l'on impose à $V_{s}$ de ne pas produire de déplacement d'énergie pour deux particules placées dans un potentiel oscillateur harmonique (Hull et Shakin) [3], soit qu'elle soit fixée de façon à obtenir une saturation au bon rayon et à la bonne énergie pour des noyaux tels que ${ }^{4} \mathrm{He}$, ${ }^{16} \mathrm{O}$ et ${ }^{40} \mathrm{Ca}$ (Abgrall) [4].

- Soit que l'on prenne un potentiel qui donne de bons résultats quand il est utilisé pour la matière nucléaire infinie (Baranger et Muthukrishnan) [5].

Les résultats, en ce qui concerne les énergies, sont les suivants (le couplage spin orbite n'a pas été inclus, sauf dans Hull et Shakin).

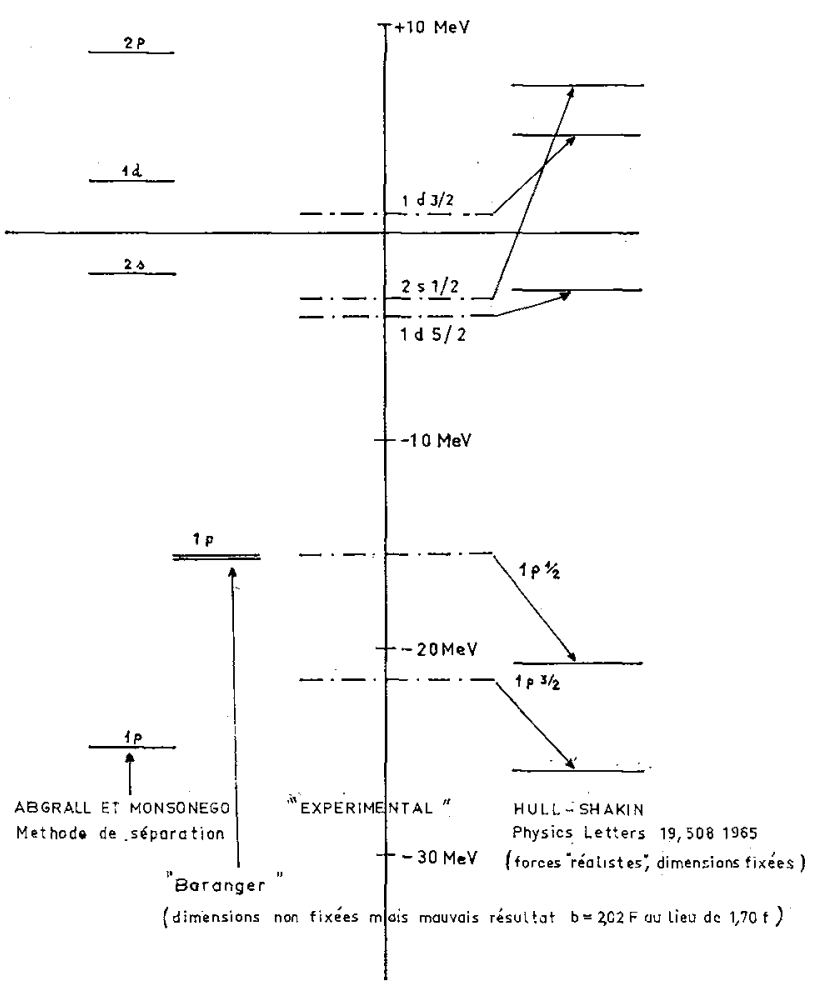

FIG. 1.

En ce qui concerne les fonctions d'onde, pour les états fortement liés, elles ne diffèrent pas beaucoup des fonctions d'onde de l'oscillateur. Développées sur cette base, elles donnent les résultats suivants :

\begin{tabular}{llllll} 
& & $R_{1 p}$ & \multicolumn{1}{c}{$R_{2 p}$} & \multicolumn{1}{c}{$R_{3 p}$} & \multicolumn{1}{c}{$R_{\mathbf{A p}_{p}}$} \\
\cline { 2 - 6 } $\mathrm{U}_{1 p}(r)$ & $\begin{array}{l}\text { Abgrall et } \\
\text { Monsonégo }\end{array}$ & 0,943 & $-0,320$ & $+0,090$ & \\
& Baranger & 0,9986 & $-0,0255$ & 0,0448 & $-0,0099$
\end{tabular}

Une dernière possibilité est d'avoir au moins un schéma cohérent entre les énergies individuelles et les fonctions d'onde individuelles, cette cohérence étant assurée par un potentiel moyen. C'est ce qu'essaie 
de faire Monsonégo à Strasbourg. Les résultats préliminaires sont les suivants : il est possible de trouver un potentiel type Woods Saxon (mais non local) donnant des énergies individuelles comparables à celles déterminées plus ou moins expérimentalement, des rayons quadratiques moyens corrects, mais alors les fonctions d'onde présentent quelques différences avec celles de l'oscillateur harmonique pour les états peu liés.

c) Choix des éléments de matrice de l'interaction nucléon-nucléon. - Ici encore on peut avoir trois positions :

- La première, essentiellement pragmatique, est de choisir un schéma bien défini pour décrire les états d'un certain noyau (ou de certains noyaux). Les éléments de matrice sont alors des paramètres que l'on détermine pour avoir le meilleur accord avec l'expérience. Le test est alors de déterminer les énergies de plus de niveaux que l'on a de paramètres. C'est cette méthode qui est utilisée par Cohen et Kurath [6] pour la couche $1 p[9 \leqslant A \leqslant 16]$. Ils supposent que la plupart des niveaux de basse énergie et de parité normale de ces noyaux ont une configuration $1 p$ (sphérique). Elle a été aussi utilisée par Brussaard, Wiechers et Glandemans [7] pour les noyaux $28<A<40$ (ils ont alors 17 éléments de matrice, car ils supposent que ${ }^{28} \mathrm{Si}$ est magique, $d_{\frac{s}{2}}$ étant complètement rempli). Cette détermination est valable pour autant que le schéma soit valable, et c'est le point faible de la méthode. Mon avis est que si les éléments de Cohen et Kurath ont quelques chances d'être valables, ceux de Brussaard en ont beaucoup moins, puisqu'ils supposent que pour $28<A<40$, les états $d_{\frac{5}{2}}$ n'interviennent pas.

- La deuxième, semi-pragmatique, est d'utiliser une force conventionnelle, avec un certain nombre de paramètres, en particulier en ce qui concerne les poids respectifs de diffếrents opérateurs d'échange.

- La troisième est d'utiliser des forces réalistes, convenablement traitées avec la méthode de séparation de Scott-Moszkowski. Cette position est celle de l'école de G. Brown et ses élèves de Copenhague (Kallio, Kolltweit, Engeland, Green). La force utilisée est encore une force simplifiée, puisqu'elle n'agit que dans l'onde relative $s$ [dans un preprint récent, Baranger a montré que ce n'était pas suffisant en ce qui concerne les éléments de matrice pour les noyaux ${ }^{\circ}$ semi-lourds, mais aucune étude n'a été faite dans ce sens pour les noyaux légers]. D'autre part, il est certain que l'interaction effective entre deux nucléons est différente de l'interaction nucléon-nucléon libre, parce que le cœur n'est pas inerte, et même si l'in- fluence statique de ce cœur peut être incorporée dans la définition de $d$, distance de séparation, on peut avoir des renormalisations non négligeables de l'interaction dues à une influence dynamique.

Bertsch [8] a par exemple montré que pour deux particules en dehors de la couche fermée ${ }^{16} \mathrm{O}$, à côté du diagramme (a)

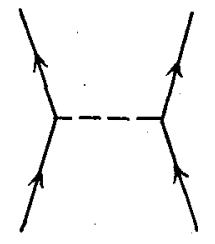

(a)

le diagramme (b) conduit à des corrections de $30 \%$.

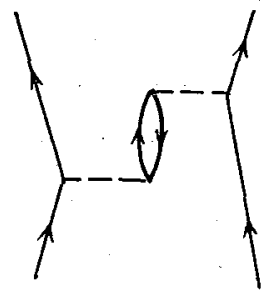

(b)

En résumé, si on n'est pas encore arrivé à utiliser de façon consistante l'interaction réelle nucléonnucléon, avec son cortège de renormalisation nécessaire, pour arriver à une interaction effective, je pense qu'on n'est pas loin de pouvoir le faire.

d) Les schémas de couplage. - $1^{\circ}$ En ce qui concerne le problème schéma $L S$-schéma $j j$, la seule pièce nouvelle à verser au dossier est l'article de Inoue et autres [9] (étude des noyaux $A=18$, $19,20)$, dont les conclusions sont les suivantes : pour les états de faible spin isobarique, la symétrie orbitale impliquée dans le modèle $L S$ est encore très importante, tandis que pour les états de plus grand spin isobarique, les deux schémas sont équivalents. Ceci est essentiellement dû au fait qu'un faible spin isobarique permet une très grande symétrie orbitale (un très grand nombre de paires de nucléons dans des états symétriques, et l'interaction est très attractive dans ce cas), tandis que pour de grands spins isobariques, cette symétrie est brisée et qu'importe ce qu'il en reste.

Par exemple, pour $A=20$, la symétrie orbitale étant indiquée par la partition correspondante :

$1^{\circ}$ (4) 6 paires symétriques, 0 paire antisymétrique

$2^{\circ}(3,1)$

$3^{\circ}(2,2)$

$4^{\circ}(211)$

4 - $-\quad, 2$ -

$5^{\circ}(1$ 111)

3 -,$- 3-1$

2. -

0 
Les éléments de matrice du couplage spin orbite l.s sont de l'ordre $\frac{1}{2}-1$ par rapport à ceux d'une paire symétrique, conduisant à un fort mélange en ce qui concerne $(2,3$ et 4$)$, mais beaucoup plus faible en ce qui concerne 1 .

$2^{\circ}$ Si l'on suppose que le schéma $L S$ garde une validité, on peut aller plus loin, et sélectionner certaines fonctions d'onde suivant un principe de symétrie supplémentaire (en l'occurrence $S U_{3}$ ), pour les états faiblement excités. Ce schéma a été, et est en vogue, parce que de ce schéma sort naturellement un spectre de rotation effectivement observé entre $A=16$ et $A=28$, et aussi parce que les fonctions d'onde calculées sans idées préconçues ressemblent beaucoup à celles que l'on a en supposant a priori un schéma $\mathrm{SU}_{3}$.

En ce qui concerne la justification de $S U_{3}$ (si justification est nécessaire), la situation est encore assez trouble, parce que, si l'on a cru longtemps que $S U_{3}$ étant valable parce que la partie à longue portée de l'interaction était bien représentée par une force quadrupole-quadrupole, la partie à courte portée étant absorbée par le potentiel moyen, Pandya et Green [10] ont montré que le cœur dur n'était peutêtre pas étranger à l'existence du spectre rotationnel observé. Ces auteurs partent d'une force attractive de type classique, et calculent ${ }^{20} \mathrm{Ne}$ en ajoutant une force répulsive ponctuelle (c'est-à-dire de la forme $\lambda \delta(r)$ d'intensité variable). Le spectre évolue vers une forme rotationnelle lorsque $\lambda$ augmente dans certaines limites.

Pour en revenir à $S U_{3}$, et prendre comme exemple ${ }^{20} \mathrm{Ne}$, ce qu'on fait est essentiellement de mettre quatre nucléons dans l'état (002) $\left[n_{x}=0, n_{y}=0, n_{z}=2\right]$ [représentation (80)] de l'oscillateur harmonique traité en coordonnées cartésiennes. La bande rotationnelle 0246 , etc. est alors extraite de cet état ( intrinsèque ) par projection. Le couplage spin orbite détruit $S U_{3}$, mais l'idée de mettre quatre nucléons dans le même état (qui sera un mélange de $1 d_{\frac{s}{2}} 1 d_{\frac{3}{2}}$ et $2 s_{\frac{1}{2}}$ ) peut être gardée. Comme dans $S U_{3}$, cette fonction d'onde ( intrinsèque » n'a pas de propriétés bien définies par rapport aux rotations (état ( déformé )) et les fonctions d'onde des états réels (correspondant à un moment angulaire bien défini) seront extraites par projection. C'est ce que font les gens de l'école Israélienne, le mélange $1 d_{\frac{s}{2}}$, $1 d_{\frac{3}{2}}$ et $2 s_{\frac{1}{2}}$ étant déterminé par minimisation de l'énergie de l'état intrinsèque. Il est alors possible de montrer que, dans certaines conditions, l'excitation d'une particule peut demander assez d'énergie pour que l'usage de bandes rotationnelles distinctes, sans trop de couplage entre elles, puisse être retenu, tandis que ceci n'est plus valable au-delà de $A=28$. De même, les états excités de parité paire dans ${ }^{16} \mathrm{O}$ peuvent être décrits à partir d'un schéma 4 particules, 4 trous (Ripka et Bassichis [11], Kelson [12], etc...), les 4 particules étant placées dans un certain état, analogue aux états occupés du ${ }^{20} \mathrm{Ne}$, les 4 trous creusés dans un état déformé de la couche $1 p$. Dans le schéma $S U_{3}$, ceci reviendrait à prendre 4 particules dans les états $\left(n_{x}=0, n_{y}=1, n_{z}=0\right)$ et à les porter dans les états $\left(n_{x}=0, n_{y}=0, n_{z}=2\right)$. Il est bon de noter que $n_{z}=2, n_{x}=0, n_{y}=0$ donnent pour le mélange :

$$
-0,632\left|\cdot 1 d_{\frac{5}{2}}>+0,516\right| 1 d_{\frac{3}{2}}>+0,577 \mid 2 s_{\frac{1}{2}}>
$$

alors que le mélange de Ripka est :

$$
-0,629\left|1 d_{\frac{5}{2}}>+0,388\right| 1 d_{\frac{3}{2}}>+0,674 \mid 2 s_{\frac{1}{2}}>\text {. }
$$

On voit que la différence n'est pas extraordinaire, la proportion de $d_{\frac{3}{2}}$ étant diminuée à cause du couplage spin orbite. Dans ces conditions, en utilisant une force de Rosenfeld, l'énergie d'excitation de 4 trous4 particules est assez basse pour enfin expliquer le niveau $6,06 \mathrm{MeV} 0^{+}$de ${ }^{16} \mathrm{O}$. Le rôle de la force de Rosenfeld (beaucoup de Majorana) est essentiel. On peut le voir facilement de la façon suivante : la configuration de l'oxygène-16 peut être représentée en coordonnées cartésiennes par $(000)^{4}(100)^{4}(010)^{4}$ $(001)^{4}$.

L'interaction dans chaque groupe est relativement indépendante du mélange Wigner-Majorana. Par contre, l'interaction entre groupes y est très sensible, diminuant quand Majorana augmente. II est alors concevable d'avoir assez de Majorana pour que ces liaisons soient si faibles qu'il ne coûte presque rien de prendre un groupe $(001)^{4}$ par exemple et de le porter dans $(002)^{4}$. Mais hélas (et c'est le point d'interrogation qui reste), il est impossible d'avoir alors une énergie de liaison pour le fondamental qui soit, même de loin, correcte.

Dans tous les calculs du type précédent, les états individuels entrant dans l'état intrinsèque ressemblent tous à des états que l'on aurait si le potentiel moyen (créé par le cœur) était déformé, sans que cette idée soit poussée strictement jusqu'au bout : car la justification la plus immédiate de placer 4 particules dans l'état (002) est de supposer que l'état (002) a une énergie plus faible que les autres de la couche $s d$, et ceci est réalisé par un potentiel moyen ellipsoïdal allongé. Les fonctions d'onde individuelles ne sont sans doute pas terriblement modifiées $((002)$ provenant d'un potentiel déformé donne un peu plus de $2 s$ que de $1 d$ que le (002) sphérique, ce qui va dans 
le bon sens par rapport à Ripka), mais par contre le cœur déformé peut permettre d'expliquer des transitions (par exemple dans ${ }^{17} \mathrm{O}$ et ${ }^{18} \mathrm{O}$ ) que l'on est obligé d'expliquer dans le (modèle sphérique) par une charge effective des neutrons due à la polarisation du cour.

Dans ce cadre d'idées, les pièces nouvelles les plus importantes à verser au dossier sont les suivantes :

a) Le travail de R. Présent [13], qui a montré que les diverses propriétés de ${ }^{7} \mathrm{Li}$ (moment quadrupolaire, transitions électromagnétiques, transition $\left.\beta\left({ }^{7} \mathrm{Be}\right)\right)$, ne pouvaient être expliquées que par une introduction de l'état $1 f, 2 p$ (à côté de $1 p$ ), et de $1 d$ (à côté de $1 s$ ), introduction effectivement réalisée par le potentiel déformé.

b) Le travail de Volkov [14] qui utilise une force à cœur répulsif mou (de la forme $-A \mathrm{e}^{-\beta r^{2}}+B \mathrm{e}^{-\alpha r^{2}}$ ), et étudie les états intrinsèques pour différents noyaux de la couche $1 p$. Les fonctions d'onde choisies sont celles d'un oscillateur harmonique à symétrie axiale dont les longueurs caractéristiques $a_{0}$ (parallèle à l'axe de symétrie) et $a_{1}$ (perpendiculaire à l'axe de symétrie) sont des paramètres à déterminer par minimisation de l'énergie de l'état intrinsèque. Les résultats montrent clairement que la forme ellipsoïda!e allongée est favorisée pour ${ }^{8} \mathrm{Be}$ tandis que la forme aplatie l'est pour ${ }^{12} \mathrm{C}$. L'influence de la proportion d'échange de Majorana est illustrée par la figure suivante (Fig. 2). L'analyse des déformations par un

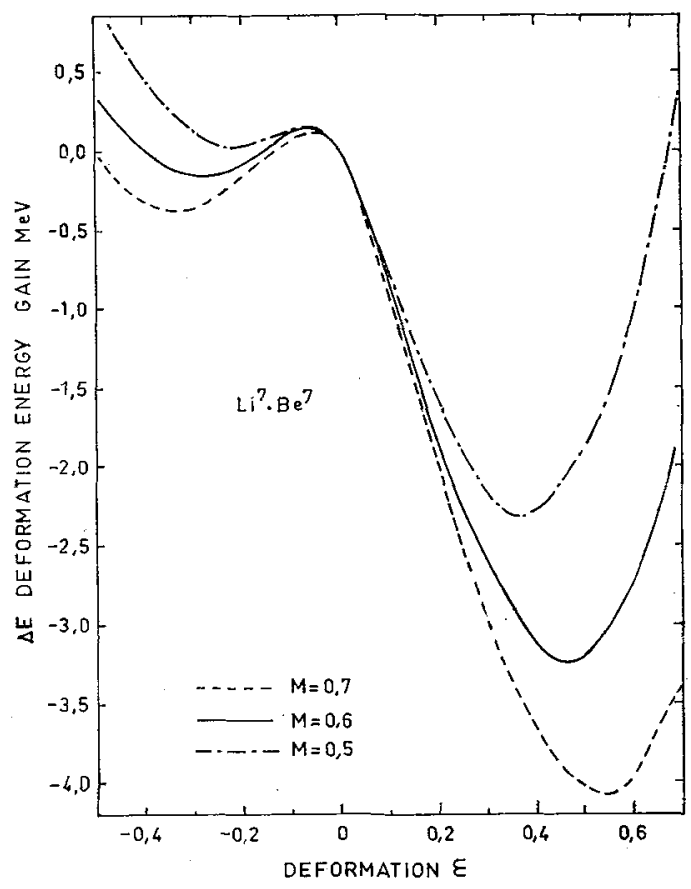

FIG. 2. schéma à la Nilsson surestime les déformations d'équilibre et les gains en énergie de la configuration déformée par rapport à la configuration sphérique.

c) A Strasbourg, nous avons étudié la même question, avec une force légèrement différente de celle utilisée par Volkov :

$$
\begin{aligned}
V=\left(-54 \mathrm{e}^{-0,25 r^{2}}+120 \mathrm{e}^{-1,5 r^{2}}\right) \\
\left(W+M P_{M}+B P_{\sigma}+H P_{\tau}\right)
\end{aligned}
$$

(énergies en $\mathrm{MeV}$, longueurs en $\mathrm{fm}$ ).

- La seule quantité qui intervienne (avec la convention $W+W=1$ ) est la quantité $1,25(W-M)+(B+H)$ qui a été fixée à $-0,333$. (Serber $(0)$, Ferrell Visscher $(-0,50)$, Rosenfeld $(-0,75) .{ }^{4} \mathrm{He}$ est approximativement reproduit :

$$
\begin{aligned}
B & =+29,6 \mathrm{MeV}, \\
\sqrt{r^{2}} & =1,48 \mathrm{fm}(\text { val. exp. } 1,41 \mathrm{fm}) .
\end{aligned}
$$

On a également pour ${ }^{16} \mathrm{O}$ :

$$
\begin{aligned}
B & =140 \mathrm{MeV}, \\
\sqrt{r^{2}} & =2,6 \text { fm (val. exp.), }
\end{aligned}
$$

pour ${ }^{40} \mathrm{Ca}$ :

$$
B=+460 \mathrm{MeV} \text { (val. exp. } 420 \mathrm{MeV} \text { ) }
$$

$$
\sqrt{r^{2}} \sim 3,29 \text { fm (val. exp. 3,52 fm)*. }
$$

Une seule configuration est utilisée (correspondant à $S U_{3}$ ), mais elle est projetée, et la minimisation est faite avant et après projection pour en étudier l'effet. Le principe de Ritz ne s'applique en fait qu'à l'état fondamental, et à un état de spin bien défini.

Les résultats sont donnés dans le tableau I.

Comme on le voit, la différence sur les paramètres de déformation peut être assez différente. (La projection favorise de plus grandes déformations). Ces résultats sont évidemment préliminaires étant donné le choix restreint des forces qui a été fait.

La force utilisée comportant peu de Majorana, l'état 4 particules- 4 trous dans ${ }^{16} \mathrm{O}$ a une trop grande énergie d'excitation. D'autre part, dans ce schéma, il est possible de reproduire le moment quadrupolaire et le $B E_{2}$ de la transition $\frac{1}{2} \rightarrow \frac{5}{2}$ dans ${ }^{17} \mathrm{O}$ sans faire appel à la notion de charge effective.

Sous une forme ou sous une autre; l'idée des états déformés a déjà permis de grands progrès dans

(*) Les énergies de liaison sont données sans corrections coulombiennes. 


\section{Tableau I}

Sphérique

$\begin{aligned}{ }^{8} \mathrm{Be} \quad B & =43 \mathrm{MeV} \\ a_{0} & =1,77 \mathrm{fm}\end{aligned}$

Sphérique

${ }^{12} \mathrm{C} \quad B=80,4 \mathrm{MeV}$

$a_{0}=1,77 \mathrm{fm}$
Avant projection

$B=49 \mathrm{MeV}(-51,5)$

$a_{0}=2,11 \mathrm{fm}$

$a_{1}=1,56 \mathrm{fm}$

Avant projection

$B=84,7 \mathrm{MeV}(85,1)$

$a_{0}=1,53 \mathrm{fm}$

$a_{1}=1,87 \mathrm{fm}$

Avant projection

${ }^{20} \mathrm{Ne}$
$B=+173,9 \mathrm{MeV}$

$a_{0}=1,92 \mathrm{fm}$

$a_{1}=1,65 \mathrm{fm}$
Après projection

$B=54,3 \mathrm{MeV}$

$a_{0}=2,32 \mathrm{fm}$

$a_{1}=1,47 \mathrm{fm}$

Après projection

$B=+87,7 \mathrm{MeV}$

$a_{0}=1,39 \mathrm{fm}$

$a_{1}=1,97 \mathrm{fm}$

Après projection

$B=+176,20 \mathrm{MeV}$

$a_{0}=2,10 \mathrm{fm}$

$a_{1}=1,61 \mathrm{fm}$ l'interprétation du spectre des noyaux légers. Ces interprétations peuvent encore différer dans leurs détails, et il y a encore beaucoup de questions non résolues, mais les figures suivantes peuvent servir d'illustration des progrès faits dans ce sens.

Sur la figure 3 , il faut remarquer l'excellent accord entre les énergies calculées et les énergies

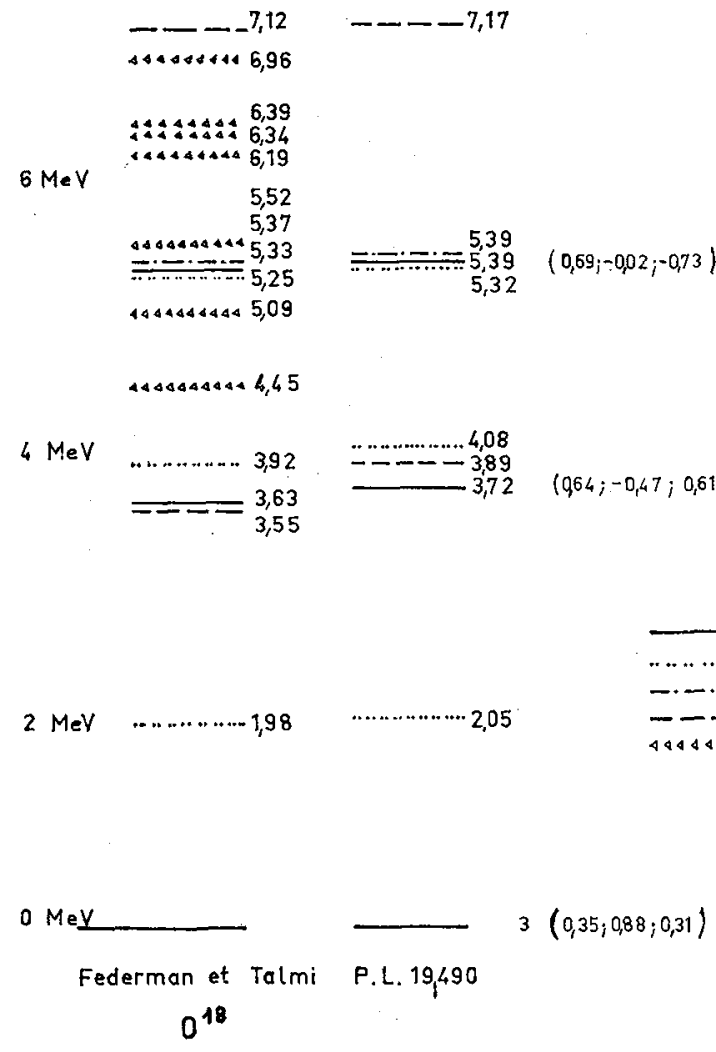

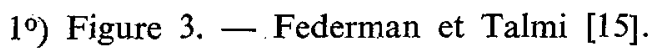

expérimentales, sur la figure 4 l'accord entre les probabilités de transitions théoriques et expérimentales.

En résumé, il me semble que les idées à suivre

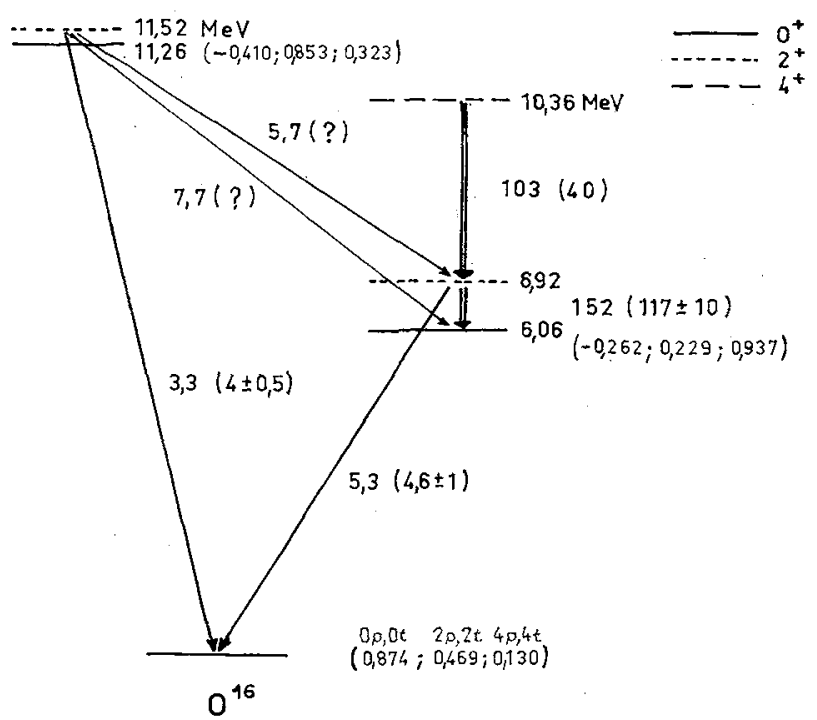

$\left.2^{\circ}\right)$ Figure 4. - G. Brown et Green [16].

dans toute la théorie des noyaux légers sont les suivantes :

a) Evaluation de l'interaction résiduelle à partir de l'interaction nucléon-nucléon libre.

b) Forme du potentiel self-consistant, et évaluation des énergies individuelles à partir des forces précédentes. 
c) Mise en évidence et interprétation des spectres à l'aide des (" états intrinsèques ) pour une fraction au moins des noyaux. A ce propos, il est nécessaire que les expérimentateurs continuent à dresser pour quelques noyaux bien choisis une carte aussi complète que possible des différentes transitions électromagnétiques, lesquelles constituent un test plus fin (en général) des fonctions d'onde utilisées.

\section{Bibliographie}

[1] Engeland (T.), Nuclear Physics, 1965, 72, 68.

[2] Kallio (A.), Kolltweir (K.), Nuclear Physics, 1964, $53,87$.

[3] Hull (M. H.), Shakin (C.), Physics Letters, 1965, 19, 506.

[4] Abgrall (Y.), Monsonégo (G.) (à paraître) Nuclear Physics.
[5] Baranger (M.), Muthukrishnan (R.), Physics Letters, 1965, 18, 160.

[6] Cohen, Kurath (O.), Nuclear Physics, 1965, 73, 1.

[7] Glandemans, Wiechers et BrussaArd, Nuclear Physics, 1964, 56, 529-548.

[8] Bertsch (G. F.), Nuclear Physics, 1965, 74, 234.

[9] Inoue (T.), Sebe (T.), Hagrwara (H.), Arima (A.), Nuclear Physics, 1964, 59, 1.

[10] Pandya (S. P.) et Green (I. M.), Nuclear Physics, 1964, 57, 658 .

[11] Bassichis (H.) et RIPKA, Physics Letters, 1965, 15, 320.

[12] Kelson (1.), Physics Letters, 1965, 16, 143.

[13] Present (R.), Phys. Rev., 1965, 139 B, 300, 1965, $140 \mathrm{~B}, 1197$.

[14] Volkov (A. B.), Nuclear Physics, 1965, 74, 33.

[15] Federman et Talmi, Physics Letters, 1965, 15, 165 , $1965,19,490$.

[16] Brown (G.), Green (A. M.) (Preprint). 